Alexander Merzkirch

Thorsten Hoos

Stefan Maas

Frank Scholzen

Daniéle Waldmann

\title{
Wie genau sind unsere Energiepässe?
}

Vergleich zwischen berechneter und gemessener Endenergie in 230 Wohngebäuden in Luxemburg

Die Mitgliedsstaaten der EU sind gemäß den Richtlinien 2002/91/EG und 2010/31/EU verpflichtet, eine Berechnungsmethodik und einen Ausweis über die Gesamtenergieeffizienz von Gebäuden aufzulegen. In Luxemburg muss nach 3 Jahren der Nutzung der reale Verbrauch an Endenergie im Pass nachgetragen werden. Für 125 Einfamilienhäuser und 105 Mehrfamilienhäuser mit insgesamt 870 Wohnungen wurde der gemessene Verbrauch und der berechnete Bedarf an Endenergie verglichen. Der berechnete Wert war im Mittel bei den Einfamilienhäusern um $74 \%$, bei den Mehrfamilienhäusern um 103 \% höher als der gemessene Wert. Die Abweichungen sind umso größer, je älter das Gebäude ist. Ursachen sind Abweichungen zwischen den teils angenommenen und teils festgelegten Eingabeparameterwerten in der Berechnungsmethodik und den tatsächlich in der Realität auftretenden Werten. Empfindliche Eingabegrößen sind hierbei Innenraumtemperatur, U-Werte, Wärmebrückenberechnung und Luftwechselrate.

Member States of the European Union are obliged to present a calculation method and certificate for the energy performance for buildings according to the guidelines 2002/91/EG und 2010/31/EU. In Luxembourg it is obligatory to add the real final energy consumption to the certificate after 3 years. The measured real final energy consumptions and the calculated ones were compared for 125 single-family homes and 105 multi-family homes with 870 dwellings in total. The mean calculated values for single-family homes were $\mathbf{7 4} \%$ higher, the mean calculated values for multi-family homes $103 \%$ higher than the actual measured data. The older the buildings, the higher the deviations, as the input parameters, which were partly assumed and partly predefined, diverge between calculations and reality. Sensitive parameters of interest are the indoor room temperature, the U-values, the used assessment for thermal bridges and the air exchange rate.

\section{Einleitung}

Die EU-Richtlinien 2002/91/EG und 2010/31/EU fordern von den Mitgliedsstaaten eine Berechnungsmethodik und einen Ausweis über die Gesamtenergieeffizienz von Gebäuden. Die Ausstellung eines Energiepasses ist daher in Luxemburg seit einigen Jahren bei Neubauten, bei Besitzer- und Mieterwechsel oder bei großen Renovierungen und Erweiterungen des Gebäudes gesetzlich verpflichtend [1]. Die Gebäude werden anhand dieser Pässe in die Kategorien von A bis I eingestuft, wobei als Kriterien dabei der Heizwärmebedarf, die Primärenergie und die zur Deckung der Primärenergie anfallende Menge an $\mathrm{CO}_{2}$ dienen. Die Endenergie wird außerdem ermittelt, weil sie eine verständliche und kontrollierbare Energieform darstellt. 
Die Berechnungsmethode des luxem-burgischen Reglements für Wohngebäude ist eine stationäre Energiebilanz und stark an die EN 832 bzw. and die Energiepässe in Deutschland nach DIN V 4108-6, DIN V 4701-10 und für Nichtwohngebäude an die DIN V 18599 angelehnt.

Die Pässe sind als Vergleichs- und Beurteilungskriterium für interessierte Besitzer, Käufer oder Mieter gedacht und stellen zugleich die Basis für staatliche Zuschüsse eines Förderungsprogrammes für Niedrigenergie- (Klasse B) und Passivhäuser (Klasse A) dar.

Im Unterscheid zu Deutschland muss der reale Verbrauch an Endenergie bei Neubauten nach 3 Jahren der Nutzung im Pass nachgetragen werden und dient damit als vergleichende Größe zur berechneten Endenergie, wobei größere Abweichungen erklärt werden müssen. Bei bestehenden Gebäuden wird je nach Verfügbarkeit auch schon bei der Erstellung des Passes der reale Endenergieverbrauch eingetragen.

Seit 2009 werden Energiepässe in Luxemburg ausgestellt. Ab 2012 liegen nun immer mehr Energiepässe vor, in denen neben den Berechnungen auch Verbrauchswerte eingetragen wurden. Eine Analyse von Berechnungs- und Verbrauchswerten ist nun erstmalig möglich.

Durch Kontakte zu öffentlichen Bauträgern und Energieberatungsbüros konnten solche Pässe von 125 Einfamilienhäusern und 105 Mehrfamilienhäusern (insgesamt 870 Wohnungen) ausgewertet werden, in denen bereits der tatsächliche Endenergieverbrauch eingetragen war, wodurch die Genauigkeit der Berechnungsmethode überprüfbar wird. In den untersuchten Gebäuden kamen als Energieträger Öl, Gas für Niedertemperatur- oder Brennwertkessel und elektrischer Strom für Wärmepumpen zur Anwendung.

\section{Methodik}

Zum Vergleich zwischen den berechneten und gemessenen Werten wurde nur die Endenergie herangezogen, da nur diese messtechnisch einfach erfasst und nachgetragen werden kann. Dies geschieht durch Bildung eines Mittelwertes der über drei Jahren gelieferten Brennstoffmengen (Öl oder Gas) oder durch die Installation eines Stromzählers zur Erfassung des Verbrauches im Fall einer Wärmepumpe. Die Gebäude wurden in 4 Baualtersklassen eingeteilt, wobei als Orientierung zur Klassenbildung der Leitfaden zur Verwendung von U-Wert-Typologien im Rahmen der Energiepasserstellung für bestehende Gebäude [2] diente. Dabei wird angenommen, dass der Baustil sich ab den 70er Jahren durch den Einfluss der Energiekrise und den Einzug von isolierten, doppelverglasten Fenstern, sowie besserer Dämmwerte der Fassaden änderte. Im Jahr 1995 wurde die erste Luxemburgische Wärmeschutzverordnung eingeführt, welche die U-Werte der Gebäudehülle gesetzlich begrenzte. Natürlich sind ältere Gebäude inzwischen teilweise oder ganz renoviert, was sich sowohl in der neuen Berechnung und als auch im Verbrauch der letzten Jahre niederschlagen sollte.

Zusätzlich zur reinen Passanalyse wurden stichprobenhaft Messungen

- zur Ermittlung des U-Wertes von Fassaden mit einer kalibrierten Wärmeflussplatte,

- zu mittleren flächen- und zeitgewichteten Innenraumtemperaturen und

- zu Transmissionswärmeverlusten aufgrund von Wärmebrücken durchgeführt [3].

\section{Ergebnisse}

In Abbildung 1 ist die berechnete und gemessene mittlere Endenergie für 125 Einfamilienhäuser in vier Baujahresklassen dargestellt, wobei als Bezugsgröße die beheizte Nettogrundfläche gemäß dem 
Luxemburger Reglement gewählt wurde. Zusätzlich sind in jeder Klasse neben der Standardabweichung Konfidenzintervalle für den Mittelwert eingetragen. Die studentsche tVerteilung ermöglicht die Berechnung der Verteilung der Differenz vom Mittelwert der Stichprobe und vom wahren Mittelwert der Grundgesamtheit. Wird eine Normal-verteilung der Stichprobe angenommen, so kann in Abhängigkeit der Stichprobenanzahl z.B. mit 95\%-iger Wahrscheinlichkeit ein Konfidenzintervall berechnet werden, in dem der tatsächliche (unbekannte) Mittelwert der Grundgesamtheit der Klasse liegt [4].

Zunächst fällt auf, dass Messung und Berechnung weit auseinander liegen (über alle Klassen gemittelt bei 74\%) und dass die Abweichungen bei neueren Gebäuden abnehmen. Vor 1970 zeigen sich im Mittel jedoch gravierende Differenzen zwischen den Berechnungen und dem tatsächlichen Verbrauch, die weit außerhalb der Konfidenzintervalle liegen. Bei Gebäuden ab 1995 sind die Abweichung geringer und Unsicherheiten größer, so dass die Mittelwerte sich annähern.

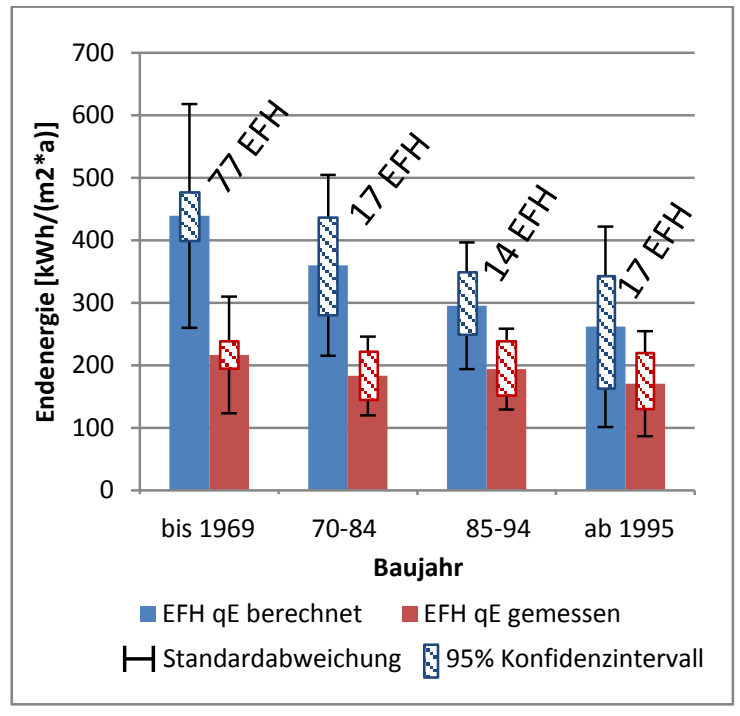

Abbildung 1: Berechnete und gemessene Endenergie qE in 125 Einfamilienhäusern (EFH) bezogen auf die Nettogrundfläche.

Fig.1: Calculated and measured final energy qE in 125 single-family homes related to the net floor area.

In Abbildung 2 sind 105 Mehrfamilienhäusern mit insgesamt 870 Wohneinheiten (WE) erfasst. Hier weicht die mittlere berechnete Endenergie über alle Klassen um $+103 \%$ vom gemessenen Verbrauch ab. Auch in diesem Fall steigt die Genauigkeit der Berechnungen mit steigendem Baujahr deutlich an. 


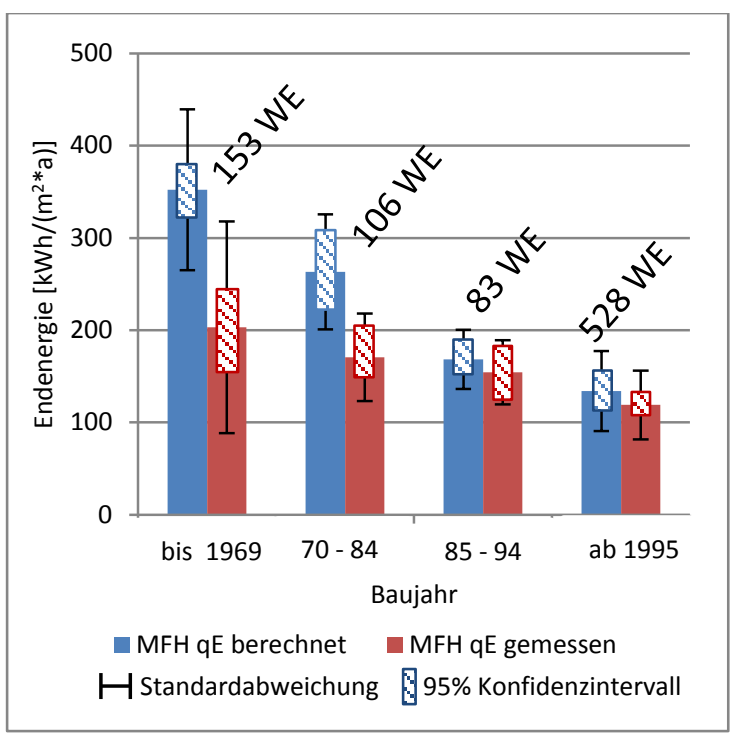

Abbildung 2: Berechnete und gemessene Endenergie qE bezogen auf die Nettogrundfläche in 870 Wohneinheiten (WE) aus insgesamt 105 Mehrfamilienhäusern.

Fig.1: Calculated and measured final energy qE in 105 single-family homes with 870 dwellings related to the net floor area.

\section{Diskussion}

Die Berechnung des Endenergieverbrauches beruht auf Eingabeparametern, von welchen einige im Reglement Grand-Ducal du 30 novembre 2007, No. 221 [1] definiert sind (Raumtemperatur), andere hingegen durch den ausstellenden Energieexperten vor Ort festgelegt werden müssen, wie z. B. UWerte, Wärmebrücken, Luftwechselrate. Betrachtet man nur die gemessenen Werte (rote Säulen) so erscheinen alte Häuser im Mittel weniger schlecht als angenommen, oder anders formuliert sind die Verbesserungen geringer als erwartet. Die neuen Häuser und Wohnungen weisen mit 170 bzw. $120 \mathrm{kWh} / \mathrm{m}^{2} / \mathrm{a}$ immer noch recht hohe Verbrauchswerte auf. Die teils gravierenden Abweichungen zwischen Berechnung und Messung beruhen auf einer hohen Sensitivität einiger Eingangsparameter, die sich durchaus mit der Bauweise ändern können.

Der immer wieder ins Spiel gebrachte Nutzereinfluss greift hier wenig, da die Fallzahlen in Abbildung 1 und 2 angegeben sind und mit insgesamt 230 Wohneinheiten ausreichend hoch sind. Maas et al [5] untersuchte den Nutzereinfluss auf den Heizenergieverbrauch in 15 Reihenhaus-siedlungen, da dort die Gebäude identisch und nur die Nutzer unterschiedlich sind. Die Standardabweichung betrug dabei etwa $1 / 3$ des Mittelwertes und das \pm Standardabweichungsintervall um den Mittelwert umfasste etwa $2 / 3$ der Häuser. Damit kann man folgern, dass in der Regel der Nutzer seinen Endenergieverbrauch um $\pm 1 / 3$ um den Mittelwert beeinflussen kann. Somit können die Abweichungen zwischen Messung und Berechnung in dieser Studie kaum über den individuellen Nutzereinfluss erklärt werden, weil dieser sich bei größeren Fallzahlen ausmitteln sollte.

In [3] wurden U-Werte von Sandstein- und Hohlblockwänden im Bestand messtechnisch ermittelt und mit den Annahmen der Baualtertypologie [2] verglichen, weil diese Annahmen in der Praxis häufig für die Erstellung der Pässe verwendet werden, ohne diese vielleicht ausreichend zu 
hinterfragen. Die Wärmeduchgangskoeffizienten oder U-Werte wurden über einen längeren Zeitraum bei ausreichender Temperaturdifferenz mit einer kalibrierten Wärmeflussplatte gemessen und ergaben für Sandsteinmauerwerke bis $1960 \mathrm{im}$ Mittel einen U-Wert von 1,1 W/m² K, während die Baualtertypologie [2] solchen Bauteilen einen Wert von 1,7 W/m $\mathrm{m}^{2} \mathrm{~K}$ zuweist, was sich mit einem Aufschlag von ca. $15 \%$ bei der Endenergie niederschlägt. Der gemessene Mittelwert für Hohlblocksteine in Gebäuden aus den 60er Jahren beträgt ebenso etwa 1,1 W/m ${ }^{2} \mathrm{~K}$ und wird in [2] mit $1,4 \mathrm{~W} / \mathrm{m}^{2} \mathrm{~K}$ angenommen, was zu etwa $10 \%$ Abweichung bei der Endenergie führt.

Als mittlere Raumtemperatur wird im Reglement $20{ }^{\circ} \mathrm{C}$ angenommen. Dadurch sollen Gebäude bei identischen Komfortverhältnissen verglichen werden können. Messungen in unsanierten Altbauten zeigen flächen- und zeitgewichtete mittlere Raumtemperaturen ab $17^{\circ} \mathrm{C}$, die dann mit zunehmender Dämmung ansteigen [3]. In Altbauten trifft häufig der Fall einer Teilbeheizung des Gebäudes zu, was natürlich den Mittelwert absenkt. Die Raumtemperatur stellt selbstverständlich eine zentrale Größe bei der Berechnung von Lüftungs- und Transmissionswärmeverlusten dar, die in diesem Baubereich mit 7-10\% pro K auf die Endenergie durchschlägt. Die in der Praxis häufig vom angenommenen Wert abweichenden Raumtemperaturen sind somit ein Grund für vom Verbrauch abweichende Berechnungen, wobei niedrigere mittlere Raumtemperaturen im Vergleich zu modernen Gebäuden auch einen geringeren Komfort bedeuten. Um eine höhere Genauigkeit bei der Berechnung der Endenergie zu erreichen, ist die Wahl der mittleren Raumtemperatur in Abhängigkeit vom U-Wert der Hülle [6] oder noch besser vom Heizwärmebedarf des Gebäudes anzustreben, was zu einer iterativen Ermittlung führen würde.

Die Luftdichtheit des Gebäudes wird im Luxemburgischen Reglement für Altbauten mit $\mathrm{n}_{50}$-Werten zwischen 4 und $8 \mathrm{~h}^{-1}$ angenommen, was dann zu Luftwechselraten von 0.7 bis $1 \mathrm{~h}^{-1}$ führt. Messungen zufolge [3] erscheint die Annahme von $n_{50}$-Werten um $4 \mathrm{~h}^{-1}$ eher angemessen, auch weil im Altbau sich der Nutzer tendenziell bei Zugerscheinungen selbst hilft und Undichtigkeiten zumindest in den sehr kalten Perioden provisorisch mit Vorhängen, Tüchern und Vorlagen abdichtet. Eine Luftwechselrate von 1 anstatt $0.7 \mathrm{~h}^{-1}$ führt zu einer Überschätzung der Lüftungsverluste und zu einem Anstieg der Endenergie um knapp 10\%. Auch hier wird offenbar die Energieeinsparung gegenüber dem Komfort priorisiert.

Nach dem Luxemburgischem Reglement können Wärmebrücken bei unsanierten Altbauten durch einen pauschalen Zuschlag von $0,1 \mathrm{~W} / \mathrm{m}^{2}$ auf den U-Wert der Hüllfläche berücksichtigt werden, was als einfache konservative Methode gedacht war. Eine detaillierte Ermittlung und Berechnung aller Wärmebrücken an vier Gebäuden führte dagegen in 3 von 4 Fällen sogar zu einer Reduktion der Transmissionsverluste [3], d.h. zu negativen Wärmebrückenkoeffizienten. Durch die außenmaßbezogene Hüllflächenermittlung und den Ansatz der eindimensionalen Wärmeleitung werden die Transmissionsverluste bereits überschätzt, so dass eine genaue Berechnung der Wärmebrücken zu negativen Korrekturen bei Außenmaßbezug führt.

Altbauten sind daher energetisch wohl besser als ihr Ruf, wenn auch mit reduziertem Komfort, wie z.B. infolge von Teilbeheizung und Zugerscheinungen. Der mittlere Endenergieverbrauch in Gebäuden bis Baujahr 1970 liegt bei nur $200 \mathrm{kWh} /\left(\mathrm{m}^{2} \mathrm{a}\right)$ und nicht wie berechnet beim Doppelten. Neubauten hingegen zeigen gegenüber Altbauten im Mittel nicht die berechneten Einsparungen oder anders formuliert nur eine geringe Abnahme beim gemessenen Verbrauch. Vor allem im Niedrigenergie- und Passivhausbereich erreichen beispielweise auch neue Schulgebäude und vor 
allem Bürogebäude in Luxemburg nicht die prognostizierten Zielwerte vor allem nicht bei der Primärenergie [7][8], so dass sich der hier angedeutete Trend umkehrt und die Berechnung zu optimistisch wird. Hier muss festgestellt werden, dass der Mittelwert für den Endenergieverbrauch bei Gebäuden ab dem Jahr 1995 für Mehrfamilienhäusern bei $120 \mathrm{kWh} /\left(\mathrm{m}^{2} a\right)$ und für Einfamilienhäusern bei $170 \mathrm{kWh} /\left(\mathrm{m}^{2} \mathrm{a}\right)$ liegt und somit nur wenig unter dem Mittelwert von Altbauten.

Die geringe Übereinstimmung zwischen Berechnung und Messung könnte auf dem Immobilienmarkt zu Diskussionen hinsichtlich der Gestaltung von Kauf- und Mietpreisen und dem Wert des Energiepasses führen.

\section{Zusammenfassung}

Die Untersuchung der Energiepässe von 230 Gebäuden zeigt, dass die Berechnung der Endenergie im Vergleich zum realen Verbrauch deutlich zu hoch ausfällt. Ist das Ziel die möglichst genaue Verbrauchsberechnung, wenn auch bei unterschiedlichem Komfort, so sind einige empfindliche energierelevante Parameter nicht praxisnah festgelegt. Im Reglement Grand-Ducal du 30 novembre 2007, No. 221 selbst ist nur die Innenraumtemperatur auf $20^{\circ} \mathrm{C}$ fixiert und die anderen diskutierten Werte sind in der Baualtertypologie [2] als Anhaltswerte angegeben, wobei deren Verwendung nicht verbindlich vorgeschrieben ist. Dennoch wird sie aus Gründen der Einfachheit oft direkt benutzt, obwohl grundsätzlich die Fachkenntnis des Experten gefragt wäre, der den Pass ausstellt. Eine Anpassung dieser Eingabegrößen würde jedoch zu einer Erhöhung der Genauigkeit bei der rechnerischen Ermittlung der Endenergie von Wohngebäuden führen.

\section{Literatur}

[1] Reglement Grand-Ducal du 30 novembre 2007, No. 221, Luxembourg.

[2] U-Wert Typologie des Ministère de l'Economie du Commerce Exterieur, 2007, Luxembourg.

[3] Hoos, T.: Einsparpotential und ökonomische Analyse der energetischen Sanierung staatlicher Gebäude in Luxemburg. Dissertation, Universität Luxembourg, 2012.

[4] Lozán, J.L.; Kausch, H.: Angewandte Statistik für Naturwissenschaftler. 3. Auflage. Hamburg, 2004.

[5] Maas, S.; Waldmann, D.; Zürbes, A.; Scheuren, J.-J.; Heinrich, H.: Der Energieverbrauch von Einfamilienhäusern in Luxemburg. In: Gesundheitsingenieur gi 129 (2008), Heft 4, S. 177232.

[6] Hens, H.; Verbeeck, G.; Verdonck, B.: Impact of energy efficiency measures on the $\mathrm{CO}_{2}$ emissions in the residential secor, a large scale analysis. In: Energy and Buildings 33 (2001) pp. 275-281.

[7] Thewes, A.: Energieeffizienz neuer Schul- und Bürogebäude in Luxemburg basierend auf Verbrauchsdaten und Simulationen. Dissertation, Universität Luxembourg 2011.

[8] Maas, S., Scholzen, F., Thewes, A., Waldmann, D., Zürbes, A.: Feldstudie zum Energieverbrauch von Bürogebäuden. Bauphysik 33 (2011), Heft 3, S. 158-166. 


\section{Autoren dieses Beitrages:}

Dipl. Wirt.-Ing. Alexander Merzkirch

Dr.-Ing. Thorsten Hoos

Prof. Dr.-Ing. Stefan Maas

Ass.-Prof. Dr.-Ing. Frank Scholzen

Ass.-Prof. Dr.-Ing. Daniéle Waldmann

Alle:

Universität Luxemburg, Campus Kirchberg

Fakultät für Naturwissenschaften, Technologie und Kommunikation, Forschungseinheit Ingenieurswissenschaften

6, Rue Richard Coudenhove Kalergi, L-1359 Luxemburg 